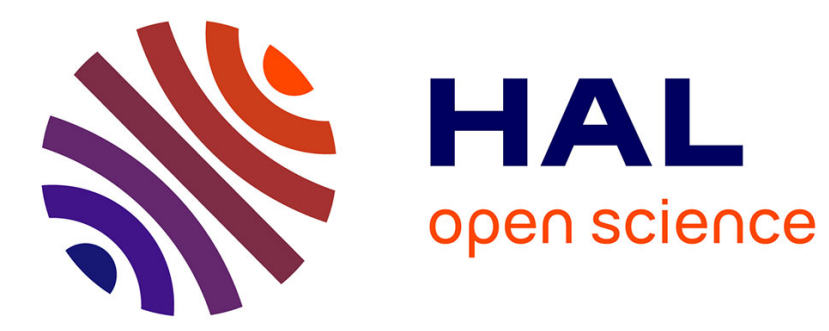

\title{
Photoelastic study of acoustic wave propagation in grain packings
}

\author{
Xavier Noblin, Guillaume Huillard, Jean Rajchenbach
}

\section{To cite this version:}

Xavier Noblin, Guillaume Huillard, Jean Rajchenbach. Photoelastic study of acoustic wave propagation in grain packings. POWDERS AND GRAINS 2009 6th International Conference on the

Micromechanics of Granular Media, Jul 2009, Golden, CO, United States. pp.439-442. hal-00455112

\section{HAL Id: hal-00455112 \\ https://hal.science/hal-00455112}

Submitted on 9 Feb 2010

HAL is a multi-disciplinary open access archive for the deposit and dissemination of scientific research documents, whether they are published or not. The documents may come from teaching and research institutions in France or abroad, or from public or private research centers.
L'archive ouverte pluridisciplinaire HAL, est destinée au dépôt et à la diffusion de documents scientifiques de niveau recherche, publiés ou non, émanant des établissements d'enseignement et de recherche français ou étrangers, des laboratoires publics ou privés. 


\title{
Photoelastic study of acoustic wave propagation in grain packings
}

\author{
Xavier Noblin, Guillaume Huillard and Jean Rajchenbach \\ Laboratoire de Physique de la Matière Condensée, UMR 6622, CNRS, Université de Nice Sophia-Antipolis, \\ Parc Valrose 06108 Nice Cedex 2, France.E-mail: xavier.noblin@unice.fr
}

\begin{abstract}
By means of photoelasticity, we success in visualizing in real time the propagation of acoustic waves in a granular packing of cylinders. As previously mentioned by Nesterenko [1] for the case of spherical grains, the nonlinearity of the contact law between grains induces a dependence of the wave velocity both on its amplitude and on the confinement force. Our experimental procedure allows an access to the local state of stress of individual grains as a function of time with a good accuracy. Our results concerning the wave velocity as a function of the amplitude, and of the confinement force, are compared to theoretical predictions and to the spherical beads case.
\end{abstract}

Keywords: Granular material, mechanical wave propagation, photoelasticity, nonlinear behavior, contact law of elastic solids PACS: $45.70 .-\mathrm{n}, 62.30 .+\mathrm{d}$

\section{INTRODUCTION}

Elastic wave propagation in granular media is of considerable interest for its fundamental understanding and for applications in Engineering as in Geophysics. These waves exemplify media where nonlinearities play an important role and have consequences such as formation of solitons [1], or nonlinear static force-velocity relationship [2],[3]. Granular materials can also give insights in wave propagation in disordered media [4]-[7]. Applications are numerous in the field of shock attenuators, explosion, seismic wave propagation [8], acoustics [9]-[11] and nondestructive control. We limit here our study to 1D grain packings.

The propagation along a 1D chain has been extensively studied for more than two decades both from the theoretical and the experimental points of view [1], [12][16]. Time-dependent photoelastic measurements have been previously performed in grain pilings in order to study load transfer as a function of the grain contact angle [17], grain shape [18] and the effect of distance and pulse duration on wave speed [19]. Other experimental studies using transducer measurements focussed on the link between contact law and propagation properties, mainly in the case of spherical beads with Hertzian contact. Those experiments probed linear and nonlinear acoustic wave and solitonic-like phenomenology [13], [16]. 1D propagation studies is a prerequisite step in order to understand more complex propagation features in higher dimensions.

The propagation of elastic waves in $2 \mathrm{D}$ and $3 \mathrm{D}$ granular media have also been largely studied. Liu and Nagel [4] interpreted the complex acoustic signal they measured as the occurrence of speckles and emphasize the high sensitivity on the geometrical arrangement. Following works have enlightened multiple diffusion processes exhibited by these systems [5], [7].

Instead of using an emitter / receiver technique, we aim at accessing directly both spatial and temporal quantitative information in 2D. The 1D case is a first step toward that goal and a topic in itself.

Here we present a study concerning the propagation of a compressive wave along a 1D chain of cylinders. The high photoelastic constant of the material allows an accurate determination of the stress state within each individual grain as a function of the time, with a frame rate up to $100000 \mathrm{fps}$. We look at the dependence of the wave speed on the imposed static compressive force and we compare the results to the spherical bead case. We also study the effect of the pulse amplitude on the velocity for a given static force.

In the following we describe first the experimental setup used here (Sec. 1 ). Then we present the experimental dependence between the static confining force, the pulse amplitude and the wave velocity (Sec. 2 ). In the last section, we compare these results to those obtained with spherical beads and to the expected theoretical behavior (Sec. 3 ).

\section{EXPERIMENTAL SETUP AND METHOD}

\section{Setup}

We use a photoelastic technique to obtain quantitative information on the stress state of individual grains in 1D packings. This technique consists in positioning stressinduced birefringent material between two circular po- 


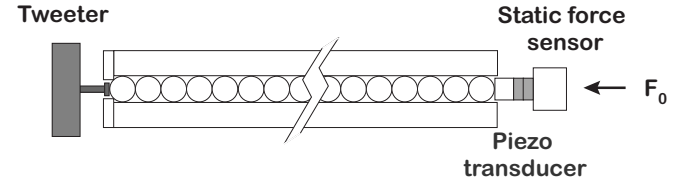

FIGURE 1. Experimental setup

larizers. Isochromatic fringes witness the state of stress in each point of photoelastic grains. We also use an interferential filter centered around $650 \mathrm{~nm}$ (width: $50 \mathrm{~nm}$ ). Grains are illuminated by means of three halogen projectors. The light is then collected by a high speed digital camera (Phantom V7.3). Successive image files are analyzed by an image analysis program written in Matlab language.

Grains are cylindrical, with diameter: $d=2 R=13 \mathrm{~mm}$ and length : $L=9.6 \mathrm{~mm}$. They are machined to this shape from a plate of PSM-1 material. Their Young's modulus is $E=2.4 G p a$ and their Poisson ratio $v=0.37$. The setup consists in a linear chain of 37 grains maintained in contact by a static force $F_{0}$ and constrained vertically (Fig. 1). A function generator driving a power amplifier allows to create a fast pulse on the moving part of a tweeter impinging the first grain of the chain. The whole chain is constrained by a piezo ceramic connected to a static force sensor which measures the static force $F_{0}$ ). These two probes aim at providing standard information concerning the static and dynamic stress values, in parallel to our direct optical measurements.

\section{The photoelastic method}

The light intensity going out from a photoelastic material located between two identical circular polarizers reads as:

$$
I=I_{0} \cos ^{2}\left[\frac{2 \pi}{f}\left(\sigma_{1}-\sigma_{2}\right)\right]
$$

Where $I_{0}$ is the maximal intensity, $\sigma_{1}$ and $\sigma_{2}$ are the local principal stresses, $f$ is the photoelastic constant of the sample. This relation holds everywhere in the material.

In order to access quantitatively to the spatio-temporal variation of pressure associated with the propagation of the acoustic pulse through the row of grains, we perform a time-resolved measurement of the magnitude of light intensity transmitted through the central region of each grains. Prior to these measurements, we carried out a calibration of the light intensity, transmitted through the central region of one grain, as a function of an applied uniaxial force. The intensity-force relationship can be expressed as:

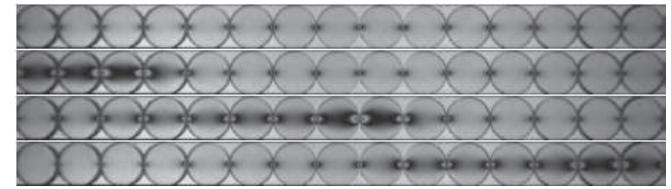

FIGURE 2. Example of four successive photoelastic images separated by $140 \mu s . F_{0}=4.5 N, F_{m}=10 N$, pulse duration: $100 \mu s$

$$
I=I_{0} \cos ^{2}\left[\frac{\pi}{2} \frac{F}{\Gamma}\right]
$$

where $F$ is the compressive force and $\Gamma$ is the force increment corresponding to the passing of the first black fringe. We find $\Gamma=20 N$. We can therefore easily access to the force $F$ by computing $F=\Gamma\left(n+\arccos \left(\frac{I}{I_{0}}\right)\right) \cdot n$ is the fringe half-order, that is $n=1 / 2$ for the first extinction and $n=1$ for the first bright fringe ( $n=0$ corresponds to zero force). To take into account residual illumination and reduced contrast, we measure the intensity at zero force: $I_{\max }=I_{0}$ and at the first extinction: $I=I_{\text {min }}$. The relation for $F$ becomes:

$$
F=\Gamma\left[n+\arccos \left(\frac{I-I_{\min }}{I_{\max }-I_{\min }}\right)\right]
$$

In Fig. 2, three successive images of a pulse propagating among some of the grains of a chain is shown.

\section{RESULTS}

We study the propagation of a compressive pulse through a chain of cylinders. Considering the mechanics of the contact between two cylinders, the relationship between the force $(F)$ and the deformation $\delta$ can be written: $F=$ $g(\delta)$. For spherical beads described by Hertz law, $g$ is a simple function of the displacement: $g(\delta) \sim \alpha \delta^{3 / 2}$. In the case of perfect cylinders, the theoretical law is more complex, and is given approximately, for the displacement, by: $\delta=\frac{2 F}{L E^{*}}\left(\ln \left(\frac{4 R L E^{*}}{F}\right)-1\right)$, with $E^{*}=\pi E /(1-$ $v^{2}$ ) [20]. Assuming that deformations occur in the contact region, the system can be seen as a spring-mass chain. We note $u_{n}$ the displacement of the grain $n$ compared to its equilibrium position. The dynamics of this system is then described by the set of equations:

$$
M \ddot{u}_{n}=g\left(\delta_{0}-u_{n}+u_{n-1}\right)+g\left(\delta_{0}-u_{n+1}+u_{n}\right)
$$

$\delta_{0}$ is the equilibrium displacement and is linked to the static force by: $F_{0}=g\left(\delta_{0}\right)$.

In the limit $\left|u_{n}-u_{n-1}\right| \ll \delta_{0}$, the system (Eq. 4) can be linearized and each contact is represented by a spring 

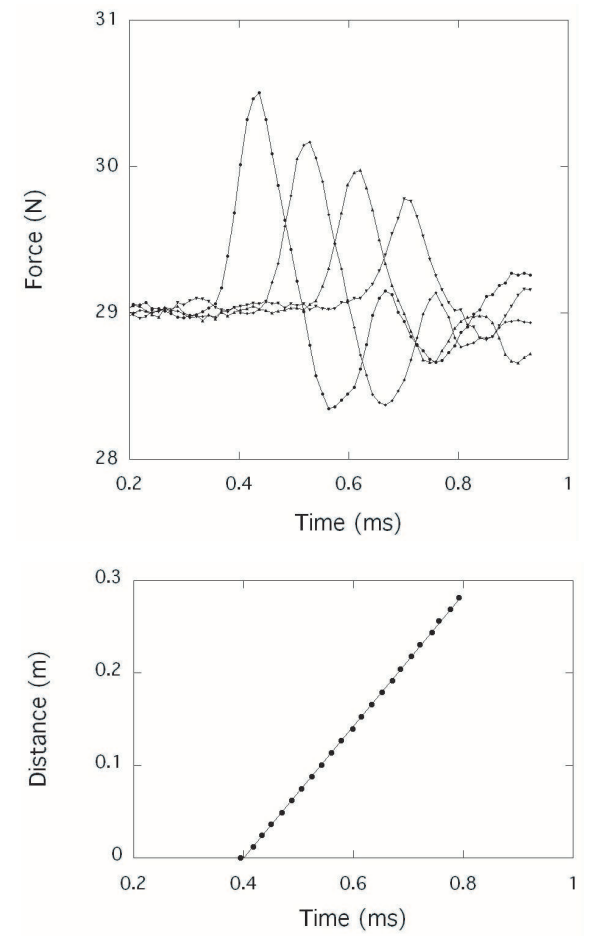

FIGURE 3. Top: dynamical force vs time for grains: 8,13 , $18,23 . F_{0}=29 N, F_{m}=2 N$. Bottom: distance vs time for the wave mid-height. The straight line is a linear fit giving $\mathrm{V}$.

of stiffness $k$. The wave equation is linear but leads to a wave velocity that depends nonlinearly on $F_{0}$.

When the dynamical displacement is of the same order or greater than $\delta_{0}$ (or alternatively when the dynamical amplitude $F_{m}$ is not small compared to the static force $\left.F_{0}\right)$, the equation is nonlinear and the wave velocity depends on the amplitude of the wave.

\section{Linear waves}

We send a $100 \mu$ s square pulse to the tweeter. The amplitude of the pulse is measured on the first grain and is noted $F_{m}$. The spatial extension of the wave corresponds to a few grains.

We first vary the static force on the chain: $F_{0}$ and keep the amplitude small compared to it. In all the experiments, $F_{m}<F_{0} / 6$, and for most of them $F_{m}<F_{0} / 10$. We can thus consider that the small deformations hypothesis holds, the wave equation is linear.

We measure the compression force on each grain as a function of time at a frame rate of $89000 \mathrm{fps}$.

In Fig. 3 (Top) we represent different curves of $F(t)$ for grain number $8,13,18,23$ as functions of time. By interpolating the time for which the wave attains its midheight, we deduce the time-distance dependence shown

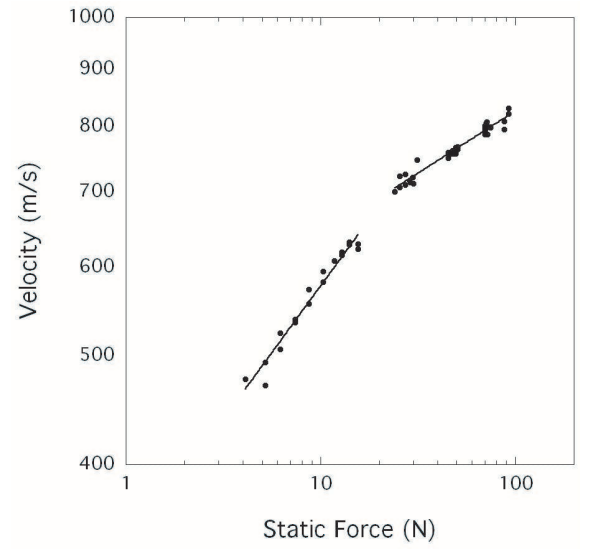

FIGURE 4. Wave speed vs. static force $F_{0}$. The straight lines are power fit with exponent 0.24 and 0.11

in Fig. 3, Bottom. A linear fit of the time-distance curve gives the wave speed $V$ (only 15 grains, in the central part of the chain, are considered for the fit). In Fig. 4 we plot the wave speed $V$ as function of $F_{0}$. We clearly see two regimes. 1) For $F_{0}<20 \mathrm{~N}$ the wave speed increases rapidly with the static force. We can measure locally an exponent close to 0.24 . This is the clear signature of a strong non linear relation at low amplitude between force and displacement. 2) For $F_{0}>20 N$, the wave speed increases in average a lot more weakly with $F_{0}$, as expected in this force range for perfect, long cylinders. A power fit gives and exponent close to 0.11 for the forcevelocity curve.

\section{Nonlinear waves}

We perform similar experiments but with $F_{0}$ constant, the maximal amplitude of the pulse is varied, with $F_{m} \geq$ $F_{0}$. In Fig. 5, we plot the wave speed as previously measured as function of $F_{m}$. We clearly see that at a given static force, the wave speed increases with the maximal force $F_{m}$. Results are very reproducible due to the higher amplitude of the signal for large pulse amplitude.

\section{DISCUSSION}

Our measurements of the wave velocity as function of the static force $F_{0}$ show for low forces $\left(F_{0}<F_{c}=20 N\right)$ a behavior similar to previous studies on the propagation of an impulse in spherical beads chains: $V \propto F_{0}^{\beta}$. Nevertheless the exponent deduced from our experiments is higher $(\beta \simeq 1 / 4)$ than that measured for beads enduring a Hertzian contact $\beta=1 / 6$. At higher forces $\left(F_{0}>F_{c}\right)$, one recovers the behavior expected for cylinders, that is 


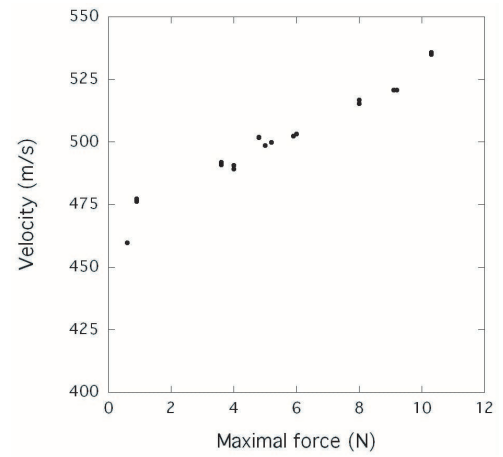

FIGURE 5. Wave speed vs. Peak amplitude $F_{m}$ for $F_{0}=$ $4.5 N$.

a very weak increase of the velocity with the confining force due to a very light nonlinearity of the contact law, the exponent $(0.11)$ is smaller than in the spherical beads case. Both regimes, below and above $F_{c}$, show very different velocity dependences versus $F_{0}$, which makes this system very different from the spherical bead case. We explain the unexpected behavior at low forces by the surface roughness of the grain, hence the microscopic nature of the contact. As mentioned by Goddard [3], conical asperities may dominate the contact stiffness below a threshold force, leading to an exponent $\beta=1 / 4$ for the velocity, close to our observations. Above the critical force, the contribution from the regular body deformation to the stiffness dominates, and the nonlinearity become lower than that for spheres, as well expected for cylinders. A broader range for $F_{0}$ and a quantitative characterization of the roughness will be achieved in a future study to a better understanding of the force-velocity relation.

\section{CONCLUSION}

We have studied the elastic wave propagation in a linear chain of cylinders using digital high speed photoelasticity. We found that for dynamic amplitude $F_{m}$ lower than the static force $F_{0}$, the force-velocity relation presents two very different regimes. Below $20 N$ the speed increases more rapidly with $F_{0}$ than for previous experiments on spheres in 1D that give an exponent $1 / 6$. Above $20 N$, the speed increases a lot more slowly with $F_{0}$. We explain this behavior by the importance of roughness at small forces, and at higher forces then the dominant effect of the cylinder-cylinder contact law which is close to be linear. In the large amplitude limit, by increasing $F_{m}$ we observe a substantial increase in the velocity. A precise study of the roughness of the cylinders surface will be carried out in order to verify quantitatively its central role in the particular force-velocity relationship.
The main consequence is that whatever the shape (cylinder, sphere...) of grains, the microstructure of the solid in contact seems to control the nonlinear behavior of the media at small forces.

\section{ACKNOWLEDGMENTS}

We would like to thank Nathalie Fraysse for a critical reading of the manuscript.

\section{REFERENCES}

1. V.F. Nesterenko, J. Appl. Mech. Tech. Phys. 24, pp. 567-575 (1983)

2. J. Duffy and R.D. Mindlin, J. Appl. Mech. 24, pp. 585-593 (1957)

3. J.D. Goddard, Proc. Roy. Soc. (London) A 430, pp. 105-131 (1990)

4. C.H. Liu and S.R. Nagel, Phys. Rev. Lett. 68, pp. 2301-2304 (1992)

5. R.L. Weaver, W. Sachse, Journal of the Acoustical Society of America 97, pp. 2094-2102 (1995)

6. X. Jia, C. Caroli and B. Velicky, Phys. Rev. Lett., 82, pp. 1863-1866 (1999)

7. X. Jia, Phys. Rev. Lett. 93, pp. 154303 (2004)

8. P.A. Johnson and X. Jia, Nature 437, pp. 871-874 (2005)

9. V. Tournat, , V.E. Gusev, and B. Castagnède, Phys. Rev. E 66, pp. $041303(2002)$

10. V. Tournat, V. Gusev, B. Castagnede. Physics Letters A 326,pp. 340-348 (2004)

11. V. Tournat, V.Y. Zaitsev, V.E. Nazarov et al. Acoustical Physics 51, pp. 543-553 (2005)

12. C. Coste, E. Falcon and S. Fauve, Phys. Rev. E 56, pp. 6104-6117 (1997)

13. C. Coste and B. Gilles, Eur. Phys. J. B 7, pp. 155-168 (1999).

14. V.F. Nesterenko, Dynamics of Heterogeneous Materials, Springer, New York (2001)

15. S. Job, F. Melo, A. Sokolow et al. Phys. Rev. Lett. 94, pp. $178002(2005)$

16. S. Job, F. Melo, A. Sokolow, S. Sen, Granular Matter 10, pp. 13-20 (2007)

17. A. Shukla, Optics and Lasers in Engineering 14, pp. 165-184 (1991)

18. A. Shukla, M. H. Sadd, R. Singh, Q. M. Tai and S. Vishwanathan, Optics and Lasers in Engineering 19, pp. 99-119 (1993)

19. A. Shukla, M. H. Sadd, Y. Xu and Q. M. Tai, J. Mech. Phys. Solids 41, pp. 1795-1808 (1993).

20. K.L. Johnson, Contact mechanics, Cambridge University Press (1992) 